\title{
La novela total o la novela fragmentaria en América Latina y los discursos de globalización y localización ${ }^{*}$
}

\author{
The total novel or the fragmented novel in Latin America \\ and the discourses of globalization and localization
}

\section{GustaVo Forero Quintero**}

Universidad de Antioquia. Medellín, Colombia

forero_fr@yahoo.fr

\section{RESUMEN}

Este texto compara dos perspectivas formales de la novela como género en la literatura hispanoamericana del siglo XX: la novela total de Mario Vargas Llosa y la novela fragmentaria de Macedonio Fernández. La primera alude al carácter absoluto del género, que da cuenta de la vida en su integridad, y la segunda a la imposibilidad del arte de hablar de la vida en todo su sentido. Desde el punto de vista del autor, cada una de ellas tiene relación con las ideologías vigentes en la contemporaneidad: la primera, con los discursos de la globalización, y la segunda, con los de la localización cultural. La novela se ve así como un género que responde de una forma u otra a la dinámica homogeneizante de la cultura o bien a su explosión discursiva, a su atomización. Ésta

* Una versión inicial de este texto fue presentada el jueves 8 de noviembre de 2008 en el marco de las XXX Jornadas Hispánicas y de América Latina: Congreso Internacional: La ciudad y los imaginarios locales en las literaturas latinoamericanas en Valparaíso, Chile. El trabajo posterior se realizó gracias al apoyo del programa de la Estrategia de Sostenibilidad del grupo GEL 2009 de la Universidad de Antioquia.

${ }^{* *}$ Profesor Asociado de la Universidad de Antioquia-Colombia. Doctor por la Universidad de Salamanca y Magíster en Études Romanes de la Universidad de la Sorbona (París IV). Dirige el Grupo Estudios Literarios (GEL). Entre sus libros están: El mito del mestizaje en la novela histórica de Germán Espinosa (Universidad Externado de Colombia, 2006), Magia de las Indias (Planeta, 2007) y la edición notada de Xicotencatl de autor anónimo (Vervuert, 2011). 
interpretación puede ser la clave difusa para la lectura de lo que hoy significa la literatura y en particular la novela.

Palabras claves: Novela, forma, literatura latinoamericana.

\section{ABSTRACT}

This paper makes a comparison between two formal perspectives of the novel as a genre in the Twentieth-Century Latin American literature: The Total Novel of Mario Vargas Llosa and the fragmented novel of Macedonio Fernández. From the autor's standpoint, each of these perspectives is related to the discourses of cultural globalization and localization, respectively. The novel is, thus, seen as a genre that responds in one way or another to the homogenizing dynamics of a certain kind of globalization or its discursive explosion, its atomization. This may be the diffuse key for reading which today means literature.

Keywords: Novel, structure, Latin American literature.

Recibido: 01/10/2010. Aceptado: 15/04/2011.

"Vargas Llosa es uno de los pocos autores que todavía cree en la literatura..." José Emilio Pacheco (Armas Marcelo, 1991: 320).

$\square$ xisten dos perspectivas formales de la novela como género literario que Etienen relación con los dos discursos políticos modernos que les sirven de telón de fondo. De una parte, se puede definir la novela moderna como una totalidad, es decir, como el género que da cuenta de una "universalidad" de perspectivas de la vida; y por otra, como aquella expresión literaria parcial y limitada que entiende la imposibilidad de representar cualquier realidad. Esta oposición se puede rastrear a través de las reflexiones de dos autores emblemáticos de la novela en América Latina: Mario Vargas Llosa (Arequipa, 28 de marzo de 1936), representante del llamado Boom latinoamericano, y Macedonio Fernández (Buenos Aires, 1874-1952), escritor vinculado con el criollismo y las vanguardias literarias de principios del siglo XX en Argentina. Ambas propuestas se entienden dentro de una reflexión formal que no responde del todo a una cronología histórica, sino que busca proponer 
una lectura estética a esa institución de la industria cultural que es hoy por hoy la literatura. Así, desde mi punto de vista, mientras la primera cree en la literatura como medio para alcanzar una descripción global del mundo, la segunda pone en duda esta capacidad y en general lo que se considera la institución misma de la literatura ${ }^{1}$. Desde el punto de vista cultural, la primera visión se puede relacionar con los discursos de la globalización, en tanto la segunda se acerca al presupuesto epistemológico de la localización cultural.

Sobre esta base se presentan, en primer lugar, los postulados de los autores respecto del género, luego su relación con el criollismo, las vanguardias y la novela del Boom Latinoamericano, para llegar a establecer los nexos entre novela, globalización y localización.

\section{LA NOVELA TOTAL Y LA NOVELA FRAGMENTARIA}

Comparando Tirant lo Blanc (1490), novela de Joanot Martorell (Gandía, 1413-Valencia, 1468), con la novela moderna ("los procedimientos y métodos de organización de la materia narrativa de Martorell anuncian casi toda la estrategia de la novela moderna" (38), dice), en Carta de batalla por Tirant lo blanc (1992) Mario Vargas Llosa señala varios elementos en común. En primer lugar, el autor de aquella obra "es el primero de esa estirpe de suplantadores de Dios -Fielding, Balzac, Dickens, Flaubert, Tolstoi, Joyce, Faulkner- que pretenden crear en sus novelas una "realidad total", el más remoto caso de novelista todopoderoso, desinteresado, omnisciente y ubicuo" (11). Desde este punto de vista, la novela respondería así a una voluntad ambiciosa y fundadora de crear un mundo. Por el contrario, en Museo de la Novela de la Eterna $(1929)^{2}$, Macedonio Fernández ataca esta capacidad del escritor contemporáneo, pues en una novela moderna ni existe una realidad, ni el principio mismo de la causalidad, y son los personajes los que actúan libremente para conformar el relato: una "novela cuyas incoherencias

${ }^{1}$ En este sentido recojo la sugerencia de Roland Barthes que en El grado cero de la escritura habla de la literatura como institución. Al respecto dice: "Toda escritura intelectual es por lo tanto el primero de los "saltos del intelecto". En vez de un lenguaje idealmente libre que no podría señalar mi persona y dejaría ignorar totalmente mi historia y mi libertad, la escritura a la que me confío es ya institución; descubre mi pasado y mi elección, me da una historia, muestra mi situación, me compromete sin que tenga que decirlo" $(1989,34)$.

${ }^{2}$ Probablemente 1929 es la fecha de escritura de la novela a pesar de que haya sido publicada de manera póstuma en 1967. El autor describe su texto como "Primera novela buena" -de un modo similar al usado también por él al referirse a su novela Adriana Buenos Aires (1938) como la "última novela mala" (150). 
de relato están zurcidas con cortes transversales que muestran lo que a cada instante hacen todos los personajes de la novela" (1995: 140). Así, frente al propósito de crear una realidad total, en este tipo de novela el escritor busca la digresión e "inaugura" lo que denomina la "literatura salteada", es decir, aquella que exige a su vez un nuevo lector "salteado" o "completo" (159) que rebase al lector tradicional de historias realistas. Su pretensión, según dice el narrador de Museo, es "confundir" (192), pues su materia es "lo inexistente" y ese lector es, a la vez, personaje o autor (174).

Desde el primer punto de vista, el escritor, como un Dios, puede abarcar una "realidad", pues, como señala Vargas Llosa refiriéndose a Martorell, "la vasta realidad fue su cantera al mismo tiempo que su paradigma. [...] porque su mirada abarca desde lo más infinitamente pequeño hasta lo más infinitamente grande" (29-30). Así, con esta pretensión, el escritor "mantiene por lo general una actitud neutral respecto de lo que cuenta" (31) y

la formidable pretensión del conjunto de la obra -imponerse como una realidad total única que a la vez es representación de la realidad total, a la que refleja ilusoriamente en sus enormidades y minucias y a todos sus niveles- tiene su réplica o equivalencia en las partes esenciales que la componen. Novela concebida a imagen y semejanza de la realidad, sus "cráteres activos" están concebidos a imagen y semejanza de la novela (38-39).

En contraposición a esto, desde la segunda perspectiva, Macedonio Fernández expresa su rechazo a esa novela que da cuenta de todo, pues, "abomino de todo realismo" (177), afirma. Para él, de manera deleznable la novela realista produce un efecto de "Alucinación", que consiste en hacerle creer al lector que está frente a la vida (174). Justamente, cree el escritor argentino, el género debe ser por naturaleza una respuesta a la lógica dominante en la modernidad y a los ecos de una cultura aferrada a la fe. De este modo, mientras para Vargas Llosa la aparición de Carmesina y el enamoramiento de Tirant es un "fragmento de realidad" que es atrapado por el escritor "en su complejidad y diversidad: en su totalidad. La narración ha integrado [allí] en una indiferenciable fluencia, en una unidad, cuatro planos, cuatro dimensiones de lo real" (un nivel retórico, uno objetivo, uno subjetivo y uno simbólico o mítico) (40-58), para Macedonio Fernández "a medida que [el autor] escrib[e], indag[a] y esper[a] sucesos, como el lector" (162); mejor:

Yo quiero que el lector sepa siempre que está leyendo una novela y no viendo un vivir, no presenciando "vida". En el momento en que el lector caiga 
en la Alucinación, ignominia del Arte, yo he perdido, no ganado lector. Lo que yo quiero es muy otra cosa, es ganarlo a él de personaje, es decir, que por un instante crea él mismo no vivir. Ésta es la emoción que me debe agradecer y que nadie pensó procurarle (174).

En tal virtud, según Fernández el escritor no hace creer al lector que está leyendo un "fragmento de la realidad", tan importante para Vargas Llosa, es decir, el escritor no crea una perspectiva de "la realidad"; por el contrario, en la novela el escritor invita a construir el propio discurso del lector que estará siempre por fuera del texto. De este modo, Macedonio Fernández busca procurar una emoción que rebase lo que se considera realidad y de ahí su crítica al automatismo de lo que llama la alucinación. La novela total sería, en esta dinámica, paradójica y ambiciosa, pero sobre todo artificial: trata de abarcar en una representación puntual la supuesta realidad en todos sus niveles, pues, según Vargas Llosa, da cuenta del mundo exterior e interior o mental, que son las modalidades absolutas de esa realidad. Este ejercicio racional sería imposible para Fernández, pues la realidad misma como tal no existe. El mundo tiene tantas representaciones como lectores pueda tener una novela y el sofisma de que existe una descripción fidedigna del mundo es sólo un artilugio. Desde su punto de vista, veríamos sólo como impostura del escritor aquello a lo que se refiere Vargas Llosa cuando advierte:

Una "novela total". Novela de caballería, fantástica, histórica, militar, social, erótica, psicológica: todas esas cosas a la vez y ninguna de ellas exclusivamente, ni más ni menos que la realidad. Múltiple, admite diferentes y antagónicas lecturas y su naturaleza varía según el punto de vista que se elija para ordenar su caos (26).

Éste es el punto más importante de oposición de las dos perspectivas. Para Vargas Llosa, es en el interior de la novela y no fuera de ella donde se ordena el "caos"; su unidad procura el orden. El escritor como Dios funda ese orden. Así, en este sentido formal podemos comprender las oposiciones irresolubles entre las dos concepciones del género, que son las dos formas contemporáneas de asumirlo: entre realidad y alucinación, totalidad y fragmentación, y, en síntesis, entre orden y caos, es decir, entre la lógica o la causalidad y lo que se considera desorden o irracionalidad. De ahí que Macedonio Fernández hable de su novela como primera novela buena o "perfecta" (194) y proponga un "Belarte" o una teoría del arte aplicada a la novela, "con algo de un ensueño" (201) y de verdadera vida. A diferencia de 
Vargas Llosa, la "certeza metafísica” de su novela consiste en

establecer la nihilidad del Tiempo y el Espacio, abstracciones que nos dicen únicamente que ocurren en nosotros representaciones de escenas o hechos que en la percepción o realidad nos procuran dolor o placer y que sin embargo se dan en nuestra mente unas veces suscitando emoción o iniciaciones motrices y otras nada de esto (207).

Para el efecto, nada más ilustrativo de este belarte de un mundo ideal que la novela misma Museo de la Novela de la Eterna, concreción de la poética de Fernández:

esta novela se parece más a la vida que la novela mala o realista, es decir la novela correcta. La congruencia (identidad) de los caracteres hace encantadores a los novelistas de la novela mala o correcta: esta congruencia nunca se mostró en una novela y no la hay en la vida; son poco realistas en esto los escritores realistas; ni siquiera saben decir qué es congruencia (226).

De esta manera, percibimos la oposición irresoluble entre ambos escritores y entre ambas teorías literarias. La novela total de Vargas Llosa implica la existencia de obras monumentales que, en sus palabras, "ordenan" el supuesto “caos". Desde este punto de vista, según el escritor peruano, Carlos Fuentes, Gabriel García Márquez y Julio Cortázar hacen parte del catálogo de escritores de novela total, pues pretenden crear en sus novelas una realidad que se organiza gracias al punto de vista del autor.

No obstante lo anterior, siguiendo las pautas de Fernández, una novela como Rayuela de Julio Cortázar (Bruselas 1914-1984) escaparía a la clasificación de Vargas Llosa, no sólo por la complejidad formal sino por su propósito vanguardista de desvirtuar cualquier lectura canónica del texto: creo que, en contraposición a los postulados de Vargas Llosa, en este autor se da justamente la dinámica transgresora que se percibía en el maestro de la "Vigilia" . Como señala Fernando Rodríguez Lafuente a propósito de la novela de Macedonio Fernández (y esto podría decirse también de la novela de Cortázar):

La lengua literaria se desdobla en dos direcciones: la desconfianza sobre la capacidad de las palabras para expresar una realidad confundida, atomi-

\footnotetext{
${ }^{3}$ Hago referencia al texto No toda es vigilia la de los ojos abiertos (Gleizer, 1928).
} 
zada, múltiple y compleja, y el rechazo de la estructura lógica del discurso, pues se considera insuficiente para explicar el decurso de las cosas, de los acontecimientos, en suma, la tragedia. Si el lenguaje se rompe en infinitos modos de representación [...] la unidad queda como gesto de inútil restauración (50).

Igual se podría pensar de otros textos de Cortázar como Historias de cronopios y famas o 62/Modelo para armar. En tal sentido, Cortázar incluyó además una foto de Macedonio en "De la seriedad en los velorios" en La vuelta al día en ochenta mundos (1967: 35), uno de sus libros atípicos, y celebró y confirmó la consideración que hizo en Cuba, en 1966, Roberto Fernández Retamar al señalar la influencia de la obra de Macedonio Fernández en $R a$ yuela. $\mathrm{Al}$ respecto, Cortázar dice:

Cuánta razón tienes en una de tus intervenciones en la mesa redonda cuando presumes una posible influencia de Macedonio Fernández en mi libro. Yo me olvidé de citarlo en su momento, y lo lamento, y es una deuda que pagaré en su día. [...] Es imposible que Macedonio no haya influido en mí aunque yo no me acuerde; no es a base de recuerdos que obran las influencias más profundas (Cortázar, 2000: 1016).

De este modo, por oposición necesaria a la novela total que exalta Vargas Llosa, creo que tanto la novela de Fernández como la de Cortázar responden de una manera fragmentaria a los problemas estéticos de la literatura en la contemporaneidad. La novela ya no describe y recompone un mundo, lo anatematiza. En consecuencia, mientras para los cultores de la novela total el género cuenta con un novelista todopoderoso, desinteresado, omnisciente y ubicuo; la novela denominada fragmentaria da cuenta de la atomización misma de este presupuesto confiable: Macedonio Fernández abandona así su podio de escritor y le da al lector una cualidad creativa y configuradora de la novela ("El desorden de mi libro es el de todas las vidas y obras aparentemente ordenadas" (248)), la pluralidad de perspectivas de la individualidad en Cortázar hacen de su novela un laberinto de sentido ["Para ese lector, mon semblable, mon frère, la novela cómica (¿y qué es Ulysses?) deberá transcurrir como esos sueños en los que al margen de un acaecer trivial presentimos una carga más grave que no siempre alcanzamos a desentrañar" (Cortázar, 1980: 449)]. La explosión del discurso así como la relatividad del narrador y la anécdota hacen de las dos novelas argentinas mencionadas paradigmas de cierta novela actual, inmersa en la tensión entre la lógica y la 
irracionalidad, entre la realidad y la emoción.

Respecto de estas diferencias entre los escritores Vargas Llosa y Macedonio Fernández, me parecen dilucidadoras las oposiciones epistemológicas entre los movimientos literarios que les sirven de trasfondo a los autores y que sustentan la disparidad de perspectivas en torno a la forma en la novela.

\section{LA NOVELA DEL CRIOLLISMO, LAS VANGUARDIAS Y LA NOVELA DEL BOOM LATINOAMERICANO}

La diferencia entre las dos concepciones del valor de la novela en el mundo contemporáneo se nota, mucho más por las relaciones paradójicas de los escritores Mario Vargas Llosa y Macedonio Fernández con el contexto estético que le da origen a sus obras. Aunque el problema formal no lo había planteado como tal el criollismo, a finales del siglo XIX y principios del XX la descripción de la vida de los gauchos, de los indios y, acaso, de la población afroamericana, lo mismo que el ambiente de reflexión en torno a la forma de principios de siglo, fueron una invitación para la superación misma del realismo y, a partir de ahí, para configurar ese anhelo deconstructivista de Fernández y, posteriormente, de las vanguardias literarias, incluido en ellas Cortázar. No obstante su realismo, el criollismo representó una primera oposición a la homogeneización de la vida "moderna". Al hablar del campesino, del "huaso" o del hombre en su contexto adverso (Mariano Latorre, Horacio Quiroga y Roberto J. Payró), con su propio lenguaje, este movimiento se planteó de manera precoz, creo yo, el tema de la identidad local por oposición a los discursos dominantes. Así, ya en el campo formal, el empleo del regionalismo o el neologismo, o la recreación poética de costumbres cotidianas como la siesta y el mate y la "pavita", elementos de contenido presentes en la obra salteada de Macedonio Fernández, constituirán la herramienta primigenia para una nueva forma de entender las tensiones planetarias entre lo global y lo local. Luego, fueran dadaístas o surrealistas los autores, fuera Cortázar o Guillermo Cabrera Infante, en las vanguardias "el fragmento se transforma en el instrumento retórico", en un modelo muy diferente "del antiguo anhelo surgido en el romanticismo de presentar al escritor como un agente provocador de la modorra colectiva, un rebelde sin más causa que él mismo o la mística. [...], el fragmento atraviesa el campo de operaciones del arte contemporáneo" (Fernández, 1995: 66). En este sentido, a diferencia de alguna novela del Boom latinoamericano, la que encarna Vargas Llosa, Fuentes o García Márquez, y en general aquella que fue 
especialmente acogida en Europa (acaso por su exotismo o por ajustarse a las preocupaciones estéticas de ese continente), otra clase de escritor empieza a hablar en distintos términos de su experiencia local. La fragmentación de su discurso se opone poco a poco al discurso generalizador de una ideología, evidente ésta en la novela tradicional, sobre todo realista o histórica. Además, la discontinuidad temporal o el sentido de unidad malograda será un duro golpe no sólo contra ese realismo automático, sino además contra la certeza argumental de esa novela histórica que tanto interesa a Vargas Llosa [Conversación en La Catedral da cuenta de este propósito].

Desde este punto de vista, Macedonio Fernández preludia las novelas "posmodernas", caracterizadas por la simultaneidad temporal y la fragmentación formal; y, por la línea de algunas vanguardias, gracias a él el género empieza a constituir una respuesta a la racionalidad dominante de los textos del realismo decimonónico (incluidos sus derivados, el realismo mágico y lo real maravilloso). Desde este momento literario, paradójicamente anterior al Boom, la forma se pone a tono con los discursos contestatarios de las ideologías dominantes y se vincula cada vez más con las expresiones locales que carecen de vigencia en las mentalidades uniformadas de Occidente.

Así, al margen de una literatura canonizada por procesos editoriales originados en centros hegemónicos de poder, surgen distintas literaturas, o bien, formas literarias que buscan trascender los modelos establecidos en esos centros. Por tal razón, hoy más que nunca la novela es el campo de cruce contemporáneo entre dos aspectos literarios propios de las industrias culturales: por una parte, el de la reflexión formal y, por otra, el de la preocupación en torno al vínculo entre la literatura y las ideologías en juego.

Por lo anterior, se pueden advertir algunas relaciones intrínsecas del hecho literario con los discursos culturales y sus nexos contradictorios con ellos.

\section{NOVELA, GLOBALIZACIÓN Y LOCALIZACIÓN}

A partir del establecimiento de sus relaciones con las ideologías, contra la unidad discursiva de la racionalidad dominante o la autoridad literaria de la narración lógica, cierta novela actual pretende exponer, de una manera no sistemática y contingente, simultánea y fragmentada, discursos que rebasan el valor de cambio que caracterizaba la escritura realista. Al respecto dice Barthes (1989): "En ese momento comienzan a multiplicarse las escrituras. En adelante, cada una, la trabajada, la populista, la neutra, la hablada, se quiere el acto inicial por el que el escritor asume o rechaza su condición 
burguesa. Cada una es un intento de respuesta a una problemática órfica de la Forma moderna: la de los escritores sin Literatura" (65).

De tal modo, frente a novelas totales como las de Carlos Fuentes y Vargas Llosa, y siguiendo la vertiente de las preocupaciones de las vanguardias en torno al valor de la literatura en sociedades modernas, aparecen cada vez más y con mayor acogida novelas fragmentarias que, con el modelo de Fernández, ponen en duda incluso lo que se considera literatura. De $A$ dos ruedas (1996), del español José Machado -novela de 80 historias cortas-, hasta El barrio era una fiesta (2005), del paraguayo Mauricio Rosencof -recreación de los inefables temas del exilio y la marginalidad en el sur-, y la historia de dos pueblos de cruce de historias en Navidad y Matanza (2007), del chileno Carlos Labbé, pasando por el mundo cotidiano de Trapos al Sol (1991), del colombiano Julio Olaciregui ${ }^{4}$, y la serie de crónicas ligeras de la ciudad Luna llena en las rocas (2000), del mexicano Xavier Velasco, es interesante observar cómo en estas novelas los discursos políticos tienen resonancia en los discursos estéticos y viceversa, pero dentro de una perspectiva fragmentaria y, retomando la palabra del principio, con una voluntad atomatizadora. Aquí vemos la relación entre novela e ideologías, lo que se ha acusado desde siempre como presupuesto material del arte en América Latina.

En efecto, si Alexis de Tocqueville (1805-1859) planteaba una recurrente relación entre la anarquía y la tiranía como extremos políticos de gobierno y desgobierno y las formas de representación estéticas en América Latina, el problema de la literatura tuvo desde el siglo XX la apariencia de la oposición entre americanismo y nacionalismo, o entre nacionalismo y cosmopolitismo, o bien, entre internacionalismo y nacionalismo, o entre nacionalismo y regionalismo, entre civilización y barbarie, y entre campo y ciudad ${ }^{5}$. Más contemporáneamente, esta relación podría verse a través de la oposición entre globalización y localización y, según lo expuesto aquí, entre novela total y novela fragmentaria. En este campo se puede preguntar entonces: ¿hasta qué punto un escritor da cuenta formal de este conflicto? o ¿qué se entendería por una novela fragmentaria y, por tanto, local? Estos parecen ser los interrogantes de hoy en torno a la institución literaria de la novela, sobre todo si se plantean con el ánimo de superar el prurito formal de considerar el realismo o el historicismo como garantías neutrales del valor literario o

\footnotetext{
${ }^{4}$ Sobre el tema de la fragmentariedad en su obra, se puede consultar mi artículo "Julio Olaciregui o el eterno fuego de la voluntad creadora”, en Luz Mery Giraldo (1995).

${ }^{5}$ Sobre este tema, me parecen pertinentes las consideraciones de Noel Salomón ("Cosmopolitismo e internacionalismo"; Carlos Bosh ("Las ideologías europeístas") o Roberto Fernández Retamar ("América Latina y el trasfondo de Occidente", en Leopoldo Zea (1993).
} 
del compromiso del artista.

La necesidad de fundar o fortalecer una unidad nacional homogénea, por oposición al reconocimiento de las regiones o de entidades territoriales, tuvo desde la independencia de los países de la región y durante todo el siglo XX los más enconados debates, máxime en función del papel de la literatura como medio de cohesión social. Pero, como apunta José Luis Romero, "la unidad no existió ni antes ni después de la independencia” (Romero, 1970: 19). El discurso aglutinante, la pretensión conciliadora, resultó desde entonces un medio para los objetivos políticos, militares y económicos (o todos al tiempo, de acuerdo con las pretensiones de los gobernantes de turno):

Antes de concluir la guerra contra España, un binomio de militares -que la revolución triunfante llamará "libertadores"- proclama la necesidad de preservar la unidad hispanoamericana. [...] Pero a esas alturas semejante unión era una utopía. Hispanoamérica estaba políticamente fragmentada y resultaba imposible superar esa fragmentación, que se apoyaba en la existencia de "patrias reformadas" (Odone, 1993: 211).

En esta dinámica, las "grandes ideas" se oponían a las ideas de caudillos o gamonales que, en el mejor de los casos, representaban la identidad de una región. En este proceso también tuvo parte la literatura. Para el caso de Argentina, por ejemplo, para José Artigas:

La autonomía provincial era el principio substancial de su ideario, en defensa irrestricta del localismo que caracterizaba la heterogeneidad de regiones aglutinadas administrativamente por la metrópoli, pero que ante la explosión revolucionaria y la presión de la capital fueron adquiriendo conciencia más clara de su personalidad diferenciada (Odone, 1993: 215).

La posición de aquellos que creían y luchaban por una unidad, se oponía, pues, a aquellos que partían de la base epistemológica de la fragmentación regional. Por otra parte, quienes defendían la fragmentación regional se encontraron con el obstáculo del cosmopolitismo que se les exigía a los intelectuales y más aún a los escritores "modernos". El discurso político, económico o militar tuvo y tiene entonces especiales vasos comunicantes con los discursos estéticos de hoy, en una sinergia apenas analizable desde la óptica histórica. Aquí, sólo propongo el ejercicio teórico de plantearlo en términos

\footnotetext{
${ }^{6}$ El autor hace referencia en este caso a la "Carta de Jamaica" (1815) de Simón Bolívar.
} 
formales de totalidad/fragmentariedad y, en términos políticos, de globalización/localización, como surge de los planteamientos teóricos de Vargas Llosa y Macedonio Fernández. La novela se ve así como un género que responde de una forma u otra a la dinámica homogeneizante de cierta globalización burguesa o bien a su explosión discursiva, a su atomización. Ésta puede ser la clave difusa para la lectura de lo que hoy significa la literatura.

\section{REFERENCIAS}

Armas Marcelo, J.J. 1991. Vargas Llosa. El vicio de escribir. Bogotá: Norma. Barthes, Roland. 1989. El grado cero de la escritura y nuevos ensayos críticos. Bogotá: Siglo Veintiuno Editores.

Bolívar, Simón. 1998. "Carta de Jamaica", en Escritos fundamentales. Caracas: Monte Ávila.

Cortázar, Julio. 1967. La vuelta al día en ochenta mundos. México: Siglo Veintiuno Editores. . 1980. Rayuela. Barcelona: Bruguera. . 2000. Cartas. Buenos Aires: Alfaguara.

Fernández, Macedonio. 1995. Museo de la Novela de la Eterna. Madrid: Cátedra.

Forero Quintero, Gustavo. 1995. "Julio Olaciregui o el eterno fuego de la voluntad creadora”, en Luz Mery Giraldo (ed). Fin de siglo: narrativa colombiana. Bogotá: Centro Editorial Javeriano, pp. 265-273.

Gleizer, Manuel. 1928. No toda es vigilia la de los ojos abiertos. Papeles de Recienvenido. Buenos Aires: Cuadernos del Plata.

Labbé, Carlos. 2007. Navidad y Matanza. Cáceres: Periférica.

Machado, José. 1996. A dos ruedas. Madrid: Alfaguara.

Odone, Juan A. 1993. "Regionalismo y nacionalismo", en Leopoldo Zea (ed). América Latina en sus ideas. México: Siglo Veintiuno Editores.

Olaciregui, Julio. 1991. Trapos al Sol. Bogotá: Planeta.

Romero, José Luis. 1970. El pensamiento político de la derecha latinoamericana. Buenos Aires: Paidós.

Rosencof, Mauricio. 2005. El barrio era una fiesta. Madrid: Alfaguara.

Vargas Llosa, Mario. 1992. Carta de batalla por Tirant lo blanc. Bogotá: Seix Barral Biblioteca Breve.

Velasco, Xavier. 2000. Luna llena en las rocas. México: Cal y Arena. Zea, Leopoldo (ed). 1993. América Latina en sus ideas. México: Siglo Veintiuno Editores. 